\title{
Prediction of Host-Derived miRNAs with the Potential to Target PVY in Potato Plants
}

\author{
Muhammad S. Iqbal ${ }^{1 *}$, Muhammad N. Hafeez ${ }^{1 *}$, Javed I. Wattoo ${ }^{2}$, Arfan Ali, ${ }^{1,3}$, \\ Muhammad N. Sharif ${ }^{1}$, Bushra Rashid ${ }^{1}$, Bushra Tabassum ${ }^{1}$ and Idrees A. Nasir ${ }^{1}$
}

${ }^{1}$ Centre of Excellence in Molecular Biology, University of the Punjab, Lahore, Pakistan, ${ }^{2}$ Faculty of Life Sciences, University of Central Punjab, Lahore, Pakistan, ${ }^{3}$ Institute of Molecular Biology and Biotechnology, The University of Lahore, Lahore, Pakistan

\section{OPEN ACCESS}

Edited by:

Karen M. Vasquez,

University of Texas at Austin, USA

Reviewed by:

Francesco Russo,

University of Copenhagen, Denmark

Zhuofei Xu,

KU Leuven, Belgium

*Correspondence:

Muhammad S. lqbal

shahzad.iqbal@cemb.edu.pk

Muhammad N. Hafeez

chnadeemhfz@gmail.com

Specialty section:

This article was submitted to Bioinformatics and Computational

Biology,

a section of the journal

Frontiers in Genetics

Received: 29 March 2016

Accepted: 24 August 2016 Published: 14 September 2016

Citation:

Iqbal MS, Hafeez MN, Wattoo Jl, Ali A, Sharif MN, Rashid B, Tabassum B and Nasir IA (2016) Prediction of

Host-Derived miRNAs with the Potential to Target PVY in Potato Plants. Front. Genet. 7:159 doi: 10.3389/fgene.2016.00159
Potato virus $Y$ has emerged as a threatening problem in all potato growing areas around the globe. PVY reduces the yield and quality of potato cultivars. During the last 30 years, significant genetic changes in PVY strains have been observed with an increased incidence associated with crop damage. In the current study, computational approaches were applied to predict Potato derived miRNA targets in the PVY genome. The PVY genome is approximately 9 thousand nucleotides, which transcribes the following 6 genes:Cl, Nla, NIb-Pro, HC-Pro, CP, and VPg. A total of 343 mature miRNAs were retrieved from the miRBase database and were examined for their target sequences in PVY genes using the minimum free energy (mfe), minimum folding energy, sequence complementarity and mRNA-miRNA hybridization approaches. The identified potato miRNAs against viral mRNA targets have antiviral activities, leading to translational inhibition by mRNA cleavage and/or mRNA blockage. We found 86 miRNAs targeting the PVY genome at 151 different sites. Moreover, only 36 miRNAs potentially targeted the PVY genome at 101 loci. The $\mathrm{Cl}$ gene of the PVY genome was targeted by 32 miRNAs followed by the complementarity of 26, 19, 18, 16, and 13 miRNAs. Most importantly, we found 5 miRNAs (miR160a-5p, miR7997b, miR166c-3p, miR399h, and miR5303d) that could target the Cl, Nla, NIb-Pro, HC-Pro, CP, and VPg genes of PVY. The predicted miRNAs can be used for the development of $P V Y$-resistant potato crops in the future.

Keywords: potato virus Y, microRNA, miRanda, RNA-22, RNA hybrid, target prediction, Solanum tuberosum, potato

\section{INTRODUCTION}

$P V Y$ has emerged as one of the most alarming pathogens in potatoes around the world; it affects the yield and quality of potatoes by inducing ringspot disease (Lorenzen et al., 2006; Ali et al., 2016). In plants, $P V Y$ can be transmitted via vegetative propagation, seed tubers and aphids (Robert et al., 2000). In recent years, biotechnology has opened new horizons to combat $P V Y$ by introducing transgenic varieties of Solanum tuberosum (McCue et al., 2012). The infection cycle of a potyvirus begins when the viral particle enters the cell via a wound or during feeding by its vector aphid (Filipowicz and Hohn, 1996; Bailey-Serres, 1999).

$P V Y$ is a positive sense, single-stranded RNA virus, belong to the Potyviridae family and genus potyvirus. Its genome size is $9.7 \mathrm{~kb}$, and it has a polyadenylated tail at the $3^{\prime}$ terminus and the VPg gene at the $5^{\prime}$ terminus. The virus releases its RNA into the cell cytoplasm, and this ssRNA uses 
the host ribosomal machinery for translation (Teycheney et al., 2000). Transcription factor elF4E is present on the $5^{\prime}$ end of the viral genome and helps in translations it does in most eukaryotes (Ruffel et al., 2002). PVY expresses its genome as a single large polypeptide that cleaves into 3 virus-specific proteases (Figure 1, Glais et al., 2002). In addition to CP, HC-Pro, and VPg were also found to be covalently attached to the PVY RNA genome (Karasev and Gray, 2013). The genome is encapsulated by nearly 2000 copies of CP (Coat Protein). The complete genome is transcribed by a single Open Reading Frame (ORF), which encodes a large polyprotein of approximately 3000-3500 amino acids ultimately cleaved by 3 viral encoded proteins (P1, Hec-Pro, and NIa-Pro). Ten mature proteins are produced after cleavage (Verchot et al., 1991).

Solanum tuberosum has an active immunity in the form of miRNAs that fights against the $P V Y$ infection. This immunity is rendered by 21-24 nt small regulatory RNAs, i.e., microRNAs (miRNAs) (Brigneti et al., 1998). miRNAs are short endogenous single-stranded RNA molecules (21-24 nt) that regulate gene expression patterns in plants and animals (Rogers and Chen, 2013) and are derived from stem loop regions of nuclear transcripts or transcripts of endogenous plant loci (Pillai et al., 2007). miRNAs are usually synthesized in the form of imperfect hairpin structures (Kim, 2005). The pri-miRNA transcript is cleaved to pre-miRNA by the Dicer-like 1 (DCL1) protein of RNase type III (Mlotshwa et al., 2008) and produces a distinctive 21-nucleotide, double-stranded RNA. This duplex is further exported into the cytoplasm by HASTY and is methylated at the $3^{\prime}$ end by HEN1 (Jones-Rhoades et al., 2006). In the cytoplasm, miRNAs direct endogenous plant transcripts for translational repression or mRNA degradation in a sequence-specific manner (Valencia-Sanchez et al., 2006). The significant role of small RNAs in plants' defense against viruses has been reported in many studies (Witkos et al., 2011).

The goal of this study is to predict the most effective miRNAs that induce resistance against $P V Y$. In this article, a nonconventional miRNA-based approach was designed to assess viral resistance in potato plants. We used the most efficient bioinformatic tools for the identification of potential miRNA target sites within the PVY genome. The artificially designed miRNAs can be used to transform Solanum tuberosum to combat $P V Y$ infection.

\section{METHODOLOGY}

\section{Data Source}

Mature microRNA sequences of potato were obtained from the miRNA database http://www.mirbase.org/cgi-bin/browse.pl. The target genome sequence was retrieved from the NCBI nucleotide database (http://ncbi.nlm.nih.gov) bearing accession number AB714135.

\section{Sequence Analysis}

Genome organization, ORFs and nucleotide distribution of $P V Y$ genome were calculated through CLC Genomics Workbench v8.

\section{Target Prediction}

In silico tools often predict numerous target sites within the target sequence, and only a limited number of them have been validated experimentally. In plants, miRNA-mRNA attachment is less complicated than it is in animals and it mostly depends on a higher quality match between the target sequence and the miRNA than it does in animals (Witkos et al., 2011). Researchers have analyzed algorithms for their accuracy and efficiency, and

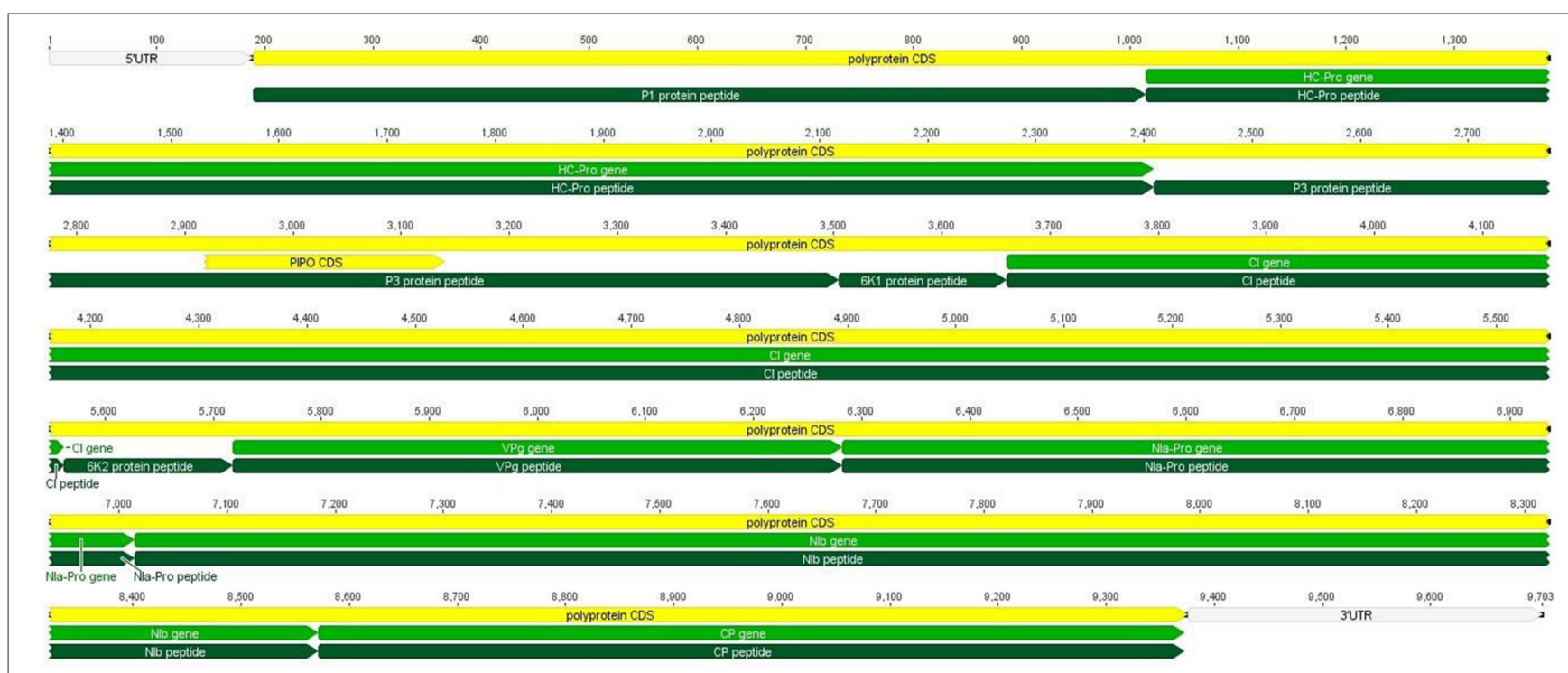

FIGURE 1 | Genome organization of PVY. Six genes (Cl, NIb, HC-Pro, Nla-Pro, VPg, CP) of PVY are shown along with their nucleotide length. The whole genome is translated as a single polypeptide. Yellow color indicates polypeptides, light green indicates specific genes and dark green represents the corresponding protein. 
some have reported their results as a guideline to be used as a stepping stone (Witkos et al., 2011). Furthermore, three different software programs were selected for the miRNA target prediction on the basis of their reported performance in the most recent literature. miRanda and RNAhybrid were among the best tools recommended for target prediction, while our third selection, RNA22, utilized a different set of analytic approaches (pattern recognition) to fully analyze the miRNA attachment with the target sequence and reduce false-positive results to some extent. Potential miRNAs targets detected are the intersection set of the results from three softwares.

\section{miRanda}

As the first miRNA target prediction software, miRanda (John et al., 2004) is the most frequently used algorithm software today for both plants and animals (John et al., 2004; Liu et al., 2015). As its script is quite basic in function for finding attachment sites, there is the possibility of producing false positives for attachment sites in the target genome. This tool selects its target match using the following three properties:(1) complimentary sequence;(2) free energy of RNA-RNA duplex and target conservation in related genomes; and (3) accounting for the final result, which is a weighted sum of the match and mismatch scores for base pairs and gap penalties. The miRanda Algorithm was downloaded from the source website (http://cbio.mskcc.org/miRNA2003/ miranda.html), and the PVY genome was assessed to determine whether there were any possible targets for Solanum tuberosum miRNAs. The analysis was performed by miRanda at its default settings $(-E=-20 \mathrm{kcal} / \mathrm{mol}$, Score threshold $=50$, energy threshold $=-20 \mathrm{kcal} / \mathrm{mol}$, scaling parameter to $z=2$, gap-open penalty to $X=-2$, gap-open penalty to $z=-8$ ).

\section{RNAhybrid}

RNAhybrid predicts miRNA and mRNA hybridization based on minimum free energy and site complementary. "Tapirhybrid," another tool rated as one of the best tools for miRNA target prediction (Srivastava et al., 2014), uses the same algorithm as RNAhybrid (as mentioned on the Tapirhybrid official webpage). RNAhybrid has also been used to locate an exact match for a miRNA target in plants (Hariharan et al., 2005). miRNAs against a $P V Y$ genome attachment were analyzed by RNAhybrid (http://bibiserv.techfak.uni-bielefeld.de/rnahybrid) (Krüger and Rehmsmeier, 2006) at an energy threshold of $-20 \mathrm{kcl} / \mathrm{mol}$, and other filters were set to the default parameters. The software reported some results of mfe that deviated from the threshold values, but these results were excluded from the final list (Table, please see Supplementary File). We used this software to eliminate any possible false positive attachments shown by miRanda. The $E$-value was set to $-20 \mathrm{kcal} / \mathrm{mol}$, and the remainder of the parameters were set to default (for more details, please see Table in Supplementary File).

\section{RNA22 v2.0 Interactive Predictions (https://cm.jefferson.edu/rna22v2/)}

The Rna22 algorithm (Miranda et al., 2006) predicts target patterns that are statistically significant miRNA motifs created after a sequence analysis of known mature miRNAs. RNA22 searches for reverse complement sites of patterns within mRNAs of interest and determines sites with many aligned patterns (socalled "hot spots"). The next step is the identification of miRNAs that are likely to bind to these sites. This approach also enables the identification of sites targeted by yet-undiscovered miRNAs. The minimum number of base-pairs between miRNA and mRNA, the maximum number of unpaired bases and the free energy cutoff were the user-defined parameters.

\section{Phylogenetic Analysis}

The complete sequence of the corresponding $P V Y$ polyprotein was retrieved from NCBI, and homologous sequences were obtained using the BLAST tool with the DELTA-BLAST (Domain Enhanced Lookup Time Accelerated Blast) algorithm referenced in protein databases. Fifty of the most homologous sequences were retrieved and aligned using the Muscle alignment tool (Edgar, 2004) along with the query sequence. Aligned sequences were used to build a phylogenetic tree using a poison algorithm by NCBI Genome Workbench.

\section{RESULTS}

Different families of miRNAs of Solanum tuberosum have considerable potential to target potato Virus-Y. miRNA families, such as miR166c-3p, miR482e-5p, miR5303a, miR5303d, miR8004, miR8032b-5p, miR8032c, miR8032e-5p, miR162b-3p, miR164-3p, miR160a-5p, miR8011a-5p, miR8018, and miR482e$5 \mathrm{p}$, were found to have more potential to target $P V Y$ at multiple loci. The $C I$ gene was targeted by 32 different miRNAs, followed by Nib, HC-Pro, NIa-Pro, $\mathrm{VPg}$, and $\mathrm{CP}$, which were targeted by 26, 19, 18, 16, and 13 miRNAs, respectively (Figure 1).

\section{miRNAs Targeting CIP (Cylindrical Inclusion Protein) Gene}

The CIP gene showed an interaction with 32 miRNAs (miR5303a, miR5303d, miR8032b-5p, miR8032c, miR8032e-5p, miR166c-3p, miR482e-5p, miR8004, miR156d-3p, miR160a-3p, miR167b-5p, miR319a-3p, miR393-3p, miR399h, miR482d-5p, miR7980a, miR8032b-3p, miR8032d-3p, miR160a-5p, miR162b-3p, miR164-3p, miR166d-5p, miR167b-3p, miR167d-3p, miR3975p, miR399l-5p, miR408a-3p, miR7996c, miR7997b, miR8018, and miR8032f-3p); Eight of these miRNAs(miR166c-3p, miR482e-5p, miR5303a, miR5303d, miR8004, miR8032b-5p, miR8032c, and miR8032e-5p) were shown to have multiple loci interactions at different nucleotide positions (Figure 2).Of note, miR166c-3p and miR482e-5p targeted the CIP gene at 7 positions, followed by miR5303a, miR5303d and miR8004, which targeted the gene at 5 positions, while miR8032b-5p, miR8032c, and miR8032e-5p targeted the gene at 3 different loci.

\section{miRNAs Targeting NIb (Nuclear Inclusion Protein b) Gene}

The NIb gene was targeted by 20 different miRNAs (miR156d-3p, miR160a-5p, miR162b-3p, miR164-3p, miR166c-3p, miR166d5p, miR167b-3p, miR167d-3p, miR169b-3p, miR169c-3p, miR5303a, miR5303d, miR7991a, miR7992-3p, miR7992-5p, 
miR7997b, miR8000, miR8006-5p, miR8011a-5p, and miR8018). It is imperative to discuss that only one miRNA (miR162b-3p) targeted the NIb gene at four different positions, while the other three miRNAs(miR164-3p, miR7992-3p, and miR8000) targeted the gene at two loci (Figure 3). The remaining16 of the 20 miRNAs that specifically targeted the Nib gene of $P V Y$ targeted the gene at a single locus.

\section{miRNAs Targeting HC-Pro (Helper Component Proteinase) Gene}

Similarly, miRNAs, such as miR164-3p, miR166c-3p, miR171a$3 p$, and miR171d-3p, targeted the HC-pro gene at multiple loci, while the gene was attacked by 19 miRNAs (Figure 5). miR164-3p targeted the HC-Pro gene at 3 different loci, and each miRNA(miR166c-3p, miR171a-3p, miR171d-3p) targeted HC-Pro at 2 different positions (Figure 4). Other than these four miRNAs, miR160a-5p, miR395a, miR395b, miR395i, miR395j, miR399h, miR399l-5p, miR408a-3p, miR482d-5p, miR8000, miR8004, miR8006-3p, miR8032b-3p, miR8032d-3p, and miR8032f-3p targeted the HC-Pro gene at a single locus.

\section{miRNAs Targeting the Nla-Pro (C-Terminal Proteinase Domain of $\mathrm{Nla}$ ) Gene}

The NIa-pro gene was potentially targeted by miR160a-5p and miR7997b at more than one locus (Figure 6). The former miRNA targeted the NIa-Pro gene at three loci, and the latter targeted at two loci. The NIa-Pro gene was observed to be attacked by 18 different miRNAs; only three of the above-mentioned
miRNAs targeted the gene at multiple loci, while the other 15 miRNAs (miR166c-3p, miR166d-5p, miR1886b, miR1886c, miR395a, miR395b, miR395i, miR395j, miR399h, miR7992-5p, miR8000, miR8006-5p, miR8007a-5p, miR8011a-5p, miR8018, and miR8033-3p) targeted at a single locus as shown in Figure 5.

\section{miRNAs Targeting(Coat Protein) and Vpg (Viral Genome-Linked Protein) Genes}

Among the 29 miRNAs targeting CP and VPg genes, only seven miRNAs targeted them at multiple loci. miR8011a$5 \mathrm{p}$ and miR8018 targeted the CP gene at six different loci (Figure 8). The other three miRNAs (miR6149-3p, miR8006$3 p$, and miR8032b-3p) targeted the CP gene at two different positions (Figure 6), and remaining eight miRNAs (miR156d-3p, miR164-3p, miR167b-3p, miR399h, miR399l-5p, miR7996c, miR8004, and miR8032d-3p) attached at a single position. While the VPg gene was targeted by miR482e-5p at three loci, the other two miRNAs (miR160a-5p and miR6149$3 \mathrm{p}$ ) targeted the VPg gene at two different loci (Figure 7). It is also important to discuss the miRNAs (miR167d3p, miR171a-3p, miR395a, miR395b, miR395i, miR395j, miR408a-3p, miR482d-5p, miR5303a, miR5303d, miR7991a, miR7997b, and miR8033-3p) that potentially targeted the $\mathrm{VPg}$ gene at a single locus (Figure 7). It is also important to mention that five miRNAs (miR160a-5p, miR7997b, miR166c-3p, miR399h, and miR5303d) could target the $C I$, NIb, HC-Pro, NIa-Pro, CP, and VPg genes of PVY at multiple loci.

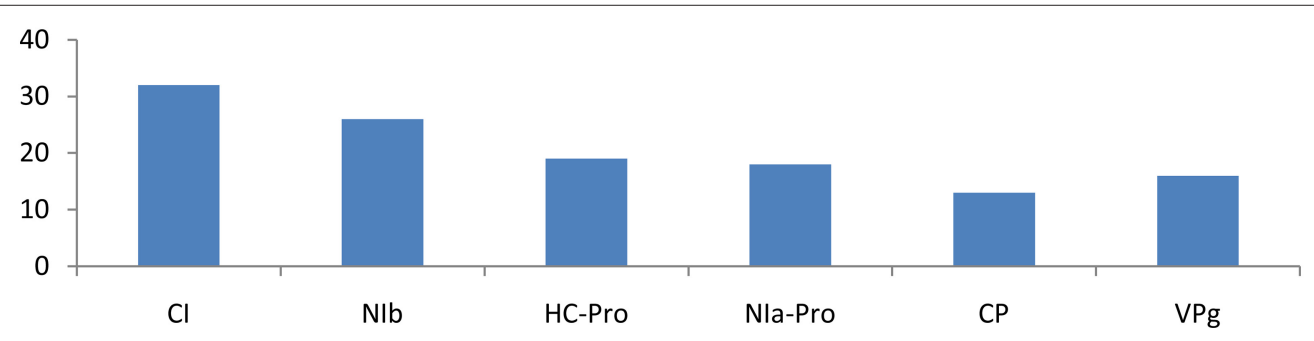

FIGURE 2 | Number of putative miRNA targets of $\mathbf{C l}, \mathbf{N} \mathbf{l b}, \boldsymbol{H C}$-Pro, $\mathbf{N l a - P r o , ~} \mathbf{P}$, and $\boldsymbol{V} \boldsymbol{P g}$ of Potato Virus $\mathbf{Y}$ (PVY). Bars show number of hits of putative miRNAs targets above threshold values calculated by various software (miRanda, RNA22, and RNAhybrid).

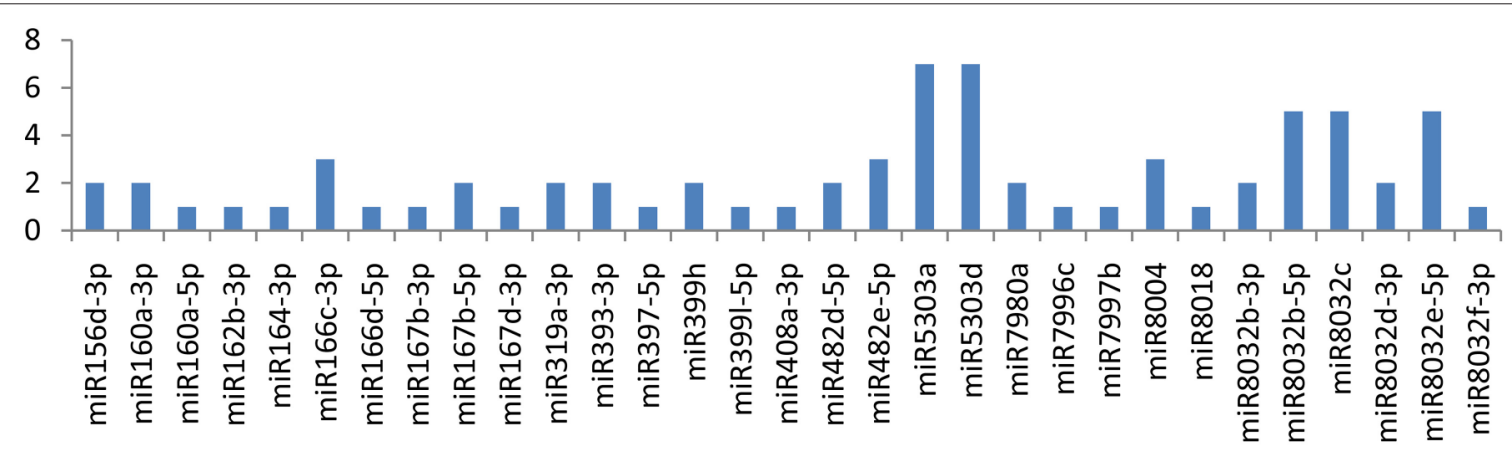

FIGURE 3 | miRNA families and the number of putative targets to the $\mathbf{C l}$ gene of thePotato Virus $\boldsymbol{Y}$ (PVY). Bars show the number of hits for individual miRNAs of Solanum tuberosum to PVY genome. 


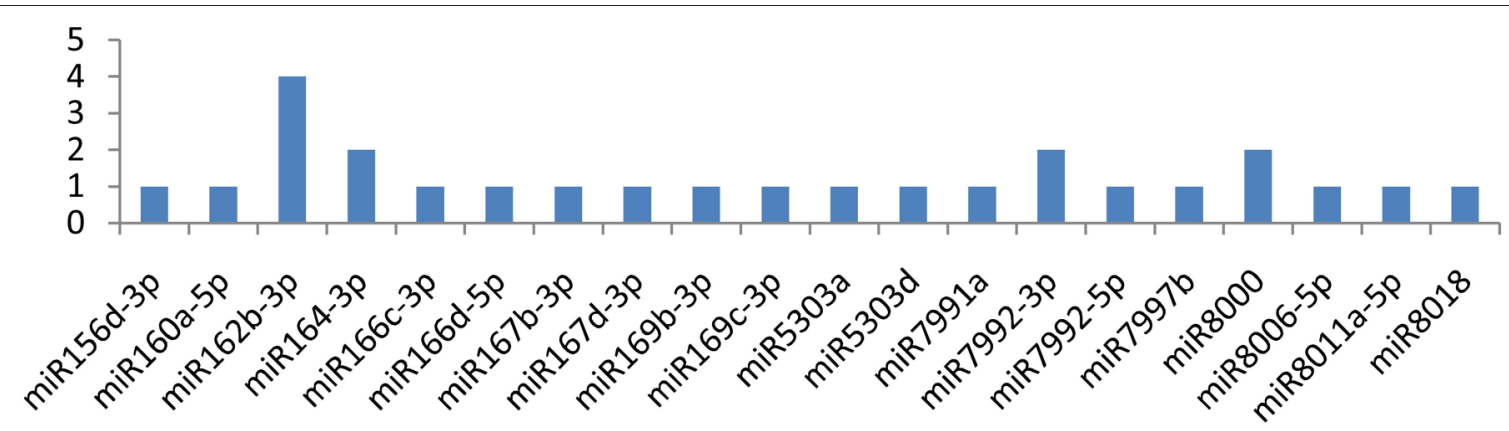

FIGURE 4 | miRNA families and the number of putative targets to the NIb gene of Potato Virus Y (PVY). Bars show the number of hits for individual miRNAs of Solanum tuberosum to PVY genome.

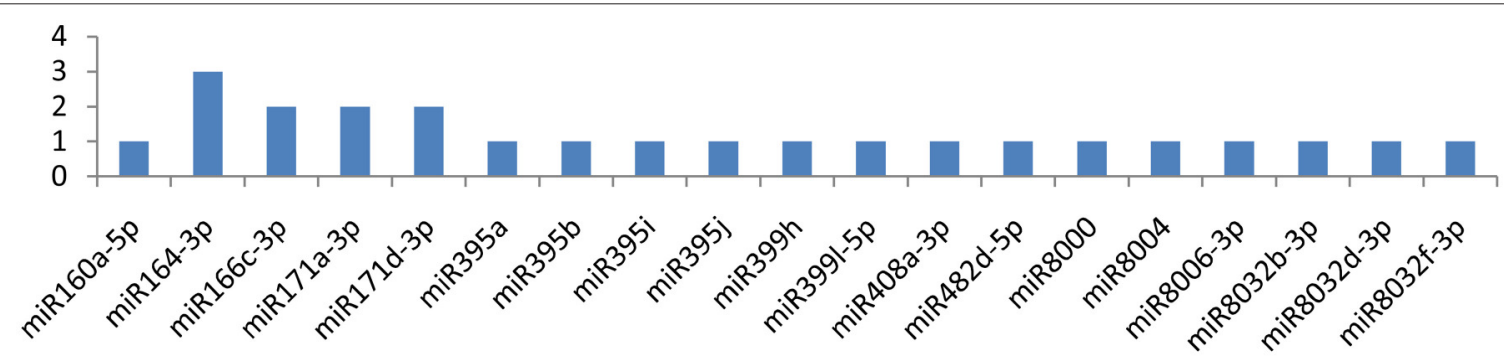

FIGURE 5 | miRNA families and the number of putative targets to the HC-Pro gene of Potato Virus Y (PVY). Bars show the number of hits for individual miRNA of Solanum tuberosum to PVY genome.

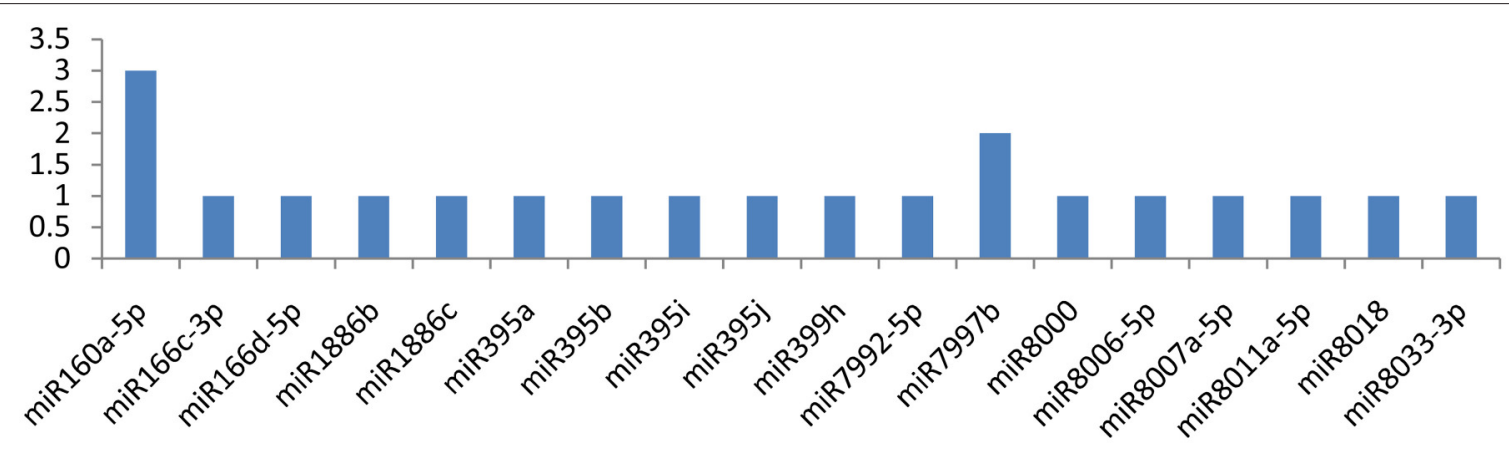

FIGURE 6 | miRNA families and the number of putative targets to the Nla-Pro gene of Potato Virus $Y(P V Y)$. Bars show the number of hits for individual miRNA of Solanum tuberosum to PVY genome.

\section{Most Effective miRNAs against Potato Virus Y (PVY)}

Using this computational approach of miRNA-mRNA nucleotide match and mismatch, we have assessed the possible off-targets of miRNAs by phylogenetically analyzing the $P V Y$ sequence. On the one hand, we have short-listed 5 miRNAs (miR160a5p, miR7997b, miR166c-3p, miR399h, and miR5303d) that could target the genes of $P V Y$ at multiple loci. On the other hand, on the basis of the polyprotein delta blast, we found other virus stains, which were genetically similar to $P V Y$ (Figure 9). This approach is helpful for planning effective experimental designs against specific or non-specific virus species.

\section{DISCUSSIONS}

The current study used bioinformatic tools to identify the miRNAs of Solanum tuberosum, which targeted $P V Y^{\mathrm{N}}$. The computational analysis concluded that 86 miRNAs targeted the PVY genome at 151 different positions, and only 36 of the 86 miRNAs targeted the CI, NIb, HC-Pro, NIa-Pro, CP, and VPg genes of $P V Y$ at 101 loci.

\section{Cylindrical Inclusion Protein}

The CI (cylindrical inclusion protein) gene was targeted by 32 miRNAs. The CI gene consists of 1901 nucleotides encoding 


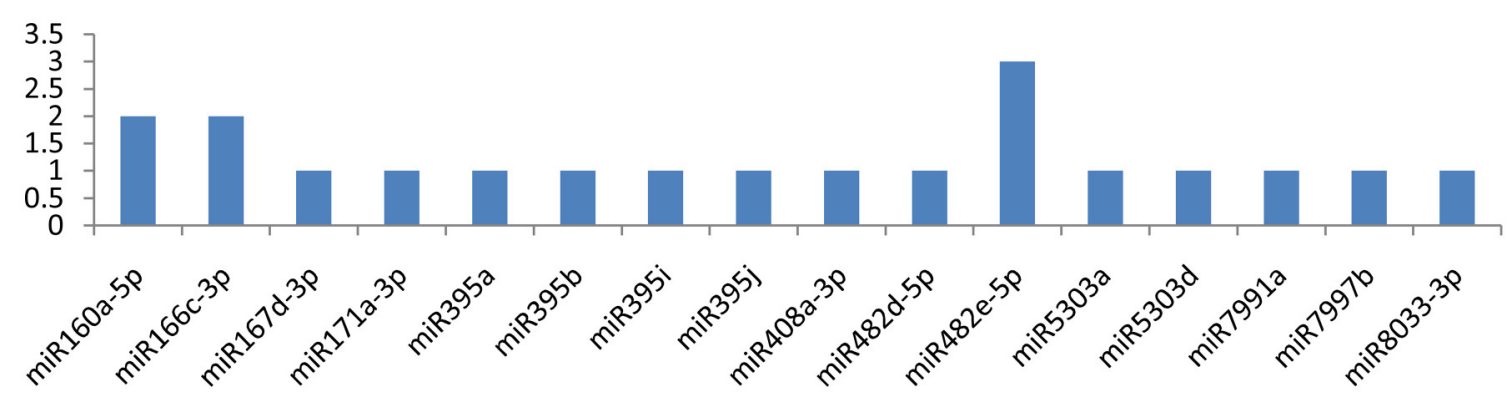

FIGURE 7 | miRNA families and the number of putative targets to the VPg gene of Potato Virus $Y$ (PVY). Bars show the number of hits for individual miRNAs of Solanum tuberosum to PVY genome.

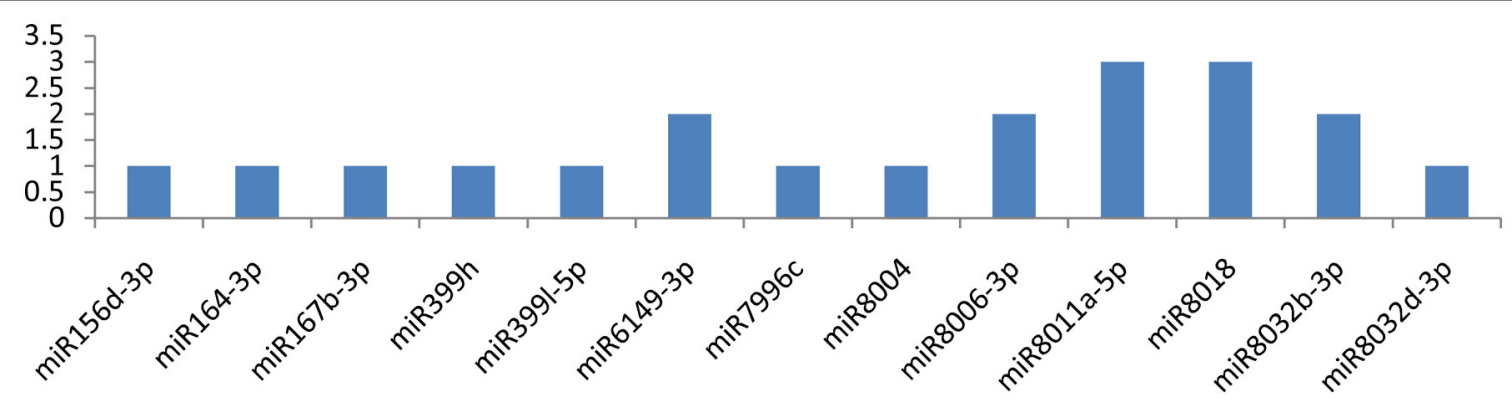

FIGURE 8 | miRNA families and the number of putative targets to the CP gene of Potato Virus $Y$ (PVY). Bars show the number of hits for individual miRNAs of Solanum tuberosum to PVY genome.

cylindrical inclusion protein. The gene has helicase activity (Lain et al., 1990; Eagles et al., 1994) and facilitates cell-tocell movement (Carrington et al., 1998). This study found 7 miRNAs, which potentially targeted the 1901-long nucleotide CI gene at multiple loci. miRNAs interfere with the replication and translation of the CI gene. A total of 32 miRNAs targeted the CI gene at 58 different positions and rendered CI functionless. The critical analysis also indicated that two miRNAs, miRNA5303a, and miRNA5303b, has great potential to target the CI gene at 14 different positions. miRNAs targeting the $C I$ gene cannot function properly, and hence, the genome of $P V Y$ remains harmless to the potato plant.

\section{Nuclear Inclusion Protein b}

The NIb (nuclear inclusion protein b) gene was targeted by 26 miRNAs, and four of the miRNAs (miR162b-3p, miR164-3p, miR7992-3p, and miR8000) were found to be more important because they targeted the gene at 10 different positions. miR162b$3 p$ potentially targeted the gene at four multiple positions, and the other three miRNAs (miR164-3p, miR7992-3p, and miR8000) targeted the gene at 6 possible sites. NIb gene nucleotides are present at the $5^{\prime}$ terminus from 7015 to 8571 nt. The gene encodes the RNA-dependent RNA polymerase, and it is involved in viral replication (Hong and Hunt, 1996). $\mathrm{NIb}$ also interacts with NIa-Pro protein, meaning that they influence each other's function or their products work in close proximity (Li et al., 1997). In addition, the interaction of NIa-pro with NIb provides a clue that NIa-pro is also involved in viral replication, probably recruiting NIb to the site of replication through a protein-protein interaction (Fellers et al., 1998). Using bioinformatic tools, we found 26 miRNAs targeting the NIa gene at 38 multiple loci, which may considerably disturb its function.

\section{Helper Component Proteinase}

In this study, we found 19 miRNAs (miR164-3p, miR166c-3p, miR171a-3p, miR171d-3p, miR160a-5p, miR395a, miR395b, miR395i, miR395j, miR399h, miR399l-5p, miR408a-3p, miR482d-5p, miR8000, miR8004, miR8006-3p, miR8032b-3p, miR8032d-3p, and miR8032f-3p) that potentially targeted the HC-Pro (helper component proteinase) gene. Among the 19 miRNAs, 4 (miR164-3p, miR166c-3p, miR171a-3p, and miR171d-3p) were found to be more critical because of their potential interaction at nine loci of the HC-Pro gene. The product of HC-Pro is proteinase (Carrington et al., 1989a,b), and similarly to Nib, it also facilitates cellto-cell transmission (Klein et al., 1994; Rojas et al., 1997), genome replication (Atreya et al., 1992; Restrepo-Hartwig and Carrington, 1994) and Aphid transmission (Pirone and Thornbury, 1984). Of note, HC-Pro is found to be a suppressor of RNA silencing (Klein et al., 1994; Kasschau and Carrington, 1998; Rovere et al., 2002). It mediates its own cleavage from polyprotein and may be considered the main gene of the viral genome, which helps the virus to escape from the plant's 


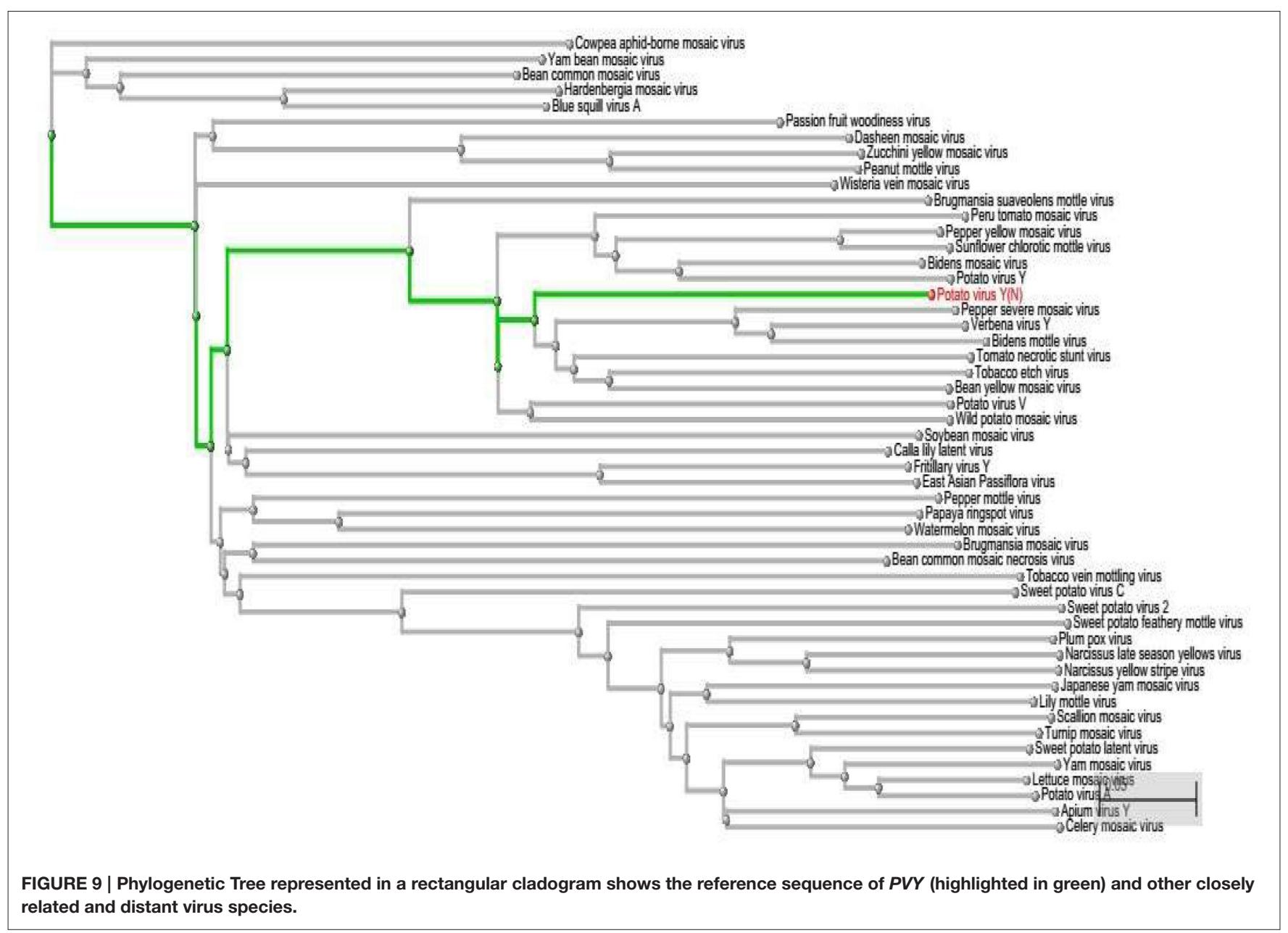

immune response (Dougherty and Carrington, 1988). We found 19 miRNAs, which targeted HC-Pro at 24 different positions, making the plant naturally resistance against $P V Y$ infection.

\section{C-Terminal Proteinase Domain of Nla}

The current bioinformatic approach revealed 18 potential miRNAs that targeted the NIa-pro (C-terminal proteinase domain of NIa) gene at multiple loci. Their potential attachment to NIa-Pro not only interferes with the normal functioning of NIa-Pro but is also responsible for the abnormal activity of Nib because both genes work in collaborative manner. We found that only two miRNAs (miR160a-5p and miR7997b) interact with NIa-pro at five different positions. The other 16 miRNAs targeted this gene at a single locus. Proteinase by nature cleaves approximately two-thirds of the viral polypeptide at multiple positions to make them functional (Riechmann et al., 1992). There are two other proteinases (P1 and HC-Pro), which play a critical role in making the viral protein functional (Shukla and Ward, 1989). A total of 18 miRNAs were found that have the potential to silence NIa proteinase, which can render it functionless.

\section{Coat Protein}

The CP (coat protein) gene was targeted by 13 miRNAs. The $\mathrm{CP}$ encodes the capsid protein, which encapsulates the singlestranded RNA genome of $P V Y$. It also facilitates cell-to-cell and long-distance movement (Dolja et al., 1995; Mahajan et al., 1996), genome amplification (Missiou et al., 2004), and Aphid transmission (Brigneti et al., 1998). Several attempts have been made to develop transgenic plants with the CP gene (Merits et al., 1998; Dan et al., 2009; Zhu et al., 2009; Tabassum et al., 2011). Using the bioinformatic approach, we found 5 miRNAs, which potentially targeted the 800 -long nucleotide $\mathrm{CP}$ gene at multiple loci. miRNAs interfere with the replication and translation of the $\mathrm{CP}$ gene and ultimately render the genome of $P V Y$ functionless. A total of 13 miRNAs targeted the $\mathrm{CP}$ gene at 20 different positions. Two microRNAs, miR8011a-5p and miR8018, were also able to target the CP gene at six different positions.

\section{Viral Genome-Linked Protein}

We found 16 miRNAs with potential to inactivate the Vpg (viral genome-linked protein) gene.

The current study revealed that miR482e-5p, miR160a-5p, and miR166c-3p cleaved the Vpg gene at 7 positions, while 
16 microRNAs targeted this gene at 20 different loci. The $V P g$ gene encodes the viral genome-linked protein, which facilitates RNA replication (Schaad et al., 1996; Rajamäki and Valkonen, 1999), cell-to-cell movement (Rajamäki and Valkonen, 1999; Léonard et al., 2000), and forms a complex with eukaryotic translation initiation factor, eIF(iso)4E (Wittmann et al., 1997; Baulcombe, 2004). We found 16 miRNAs that have the potential to interrupt the normal functioning of this gene.

Putative miRNAs targets were selected on the basis of the miRanda, RNA22, and RNAhybrid algorithm (Re). Apart from perfect complementarities in miRNAs and their targeted regions with no mismatch, up to 1-24 nucleotides were focused upon, and only un-gapped miRNA-mRNA predicted targeted models were considered as best hits. Finally, we selected only those miRNAs that were found to be potential targets by all three software.

Controlling for viral infection, following viral mRNA degradation is the simplest approach. Niu and colleagues used a 273 bp sequence of Arabidopsis miR159a per-miRNA transcript expression amiRNAs against the viral suppressor genes P69 and HC-Pro to generate resistance against Turnip yellow mosaic virus and Turnip mosaic virus infection, respectively. Here, we may use miRNAs against $P V Y$ in transgenic plants.

\section{REFERENCES}

Ali, A., Iqbal, M., Ali, Q., Razzaq, A., and Nasir, I. A. (2016). Gene profiling for invertase activity: assessment of potato varieties for resistance towards cold induced sweetening. Adv. Life Sci. 3, 63-70.

Atreya, C. D., Atreya, P. L., Thornbury, D. W., and Pirone, T. P. (1992). Site-directed mutations in the potyvirus HC-Pro gene affect helper component activity, virus accumulation, and symptom expression in infected tobacco plants. Virology 191, 106-111. doi: 10.1016/0042-6822(92) 90171-K

Bailey-Serres, J. (1999). Selective translation of cytoplasmic mRNAs in fs. Trends Plant Sci. 4, 142-148. doi: 10.1016/S1360-1385(99)01386-2

Baulcombe, D. (2004). RNA silencing in plants. Nature 431, 356-363. doi: 10.1038 /nature02874

Brigneti, G., Voinnet, O., Li, W. X., Ji, L. H., Ding, S. W., and Baulcombe, D. C. (1998). Viral pathogenicity determinants are suppressors of transgene silencing in Nicotiana benthamiana. EMBO J. 17, 6739-6746. doi: 10.1093/ emboj/17.22.6739

Carrington, J. C., Cary, S. M., Parks, T. D., and Dougherty, W. G. (1989b). A second proteinase encoded by a plant potyvirus genome. EMBO J. 8, 365 .

Carrington, J. C., Freed, D. D., and Sanders, T. C. (1989a). Autocatalytic processing of the potyvirus helper component proteinase in Escherichia coli and in vitro. J. Virol. 63, 4459-4463.

Carrington, J. C., Jensen, P. E., and Schaad, M. C. (1998). Genetic evidence for an essential role for potyvirus CI protein in cell-to-cell movement. Plant J. 14, 393-400. doi: 10.1046/j.1365-313X.1998.00120.x

Dan, L., ZhaoKui, G., XiuQing, W., PeiQiang, Y., Chan, Q., and Lei, L. (2009). RNA interference of TMV-CP and $P V Y$-CP double gene to fusion and transform tobacco. Genomics Appl. Biol. 28, 450-454.

Dolja, V. V., Haldeman-Cahill, R., Montgomery, A. E., Vandenbosch, K. A., and Carrington, J. C. (1995). Capsid protein determinants involved in cellto-cell and long distance movement of tobacco etch potyvirus. Virology 206, 1007-1016. doi: 10.1006/viro.1995.1023

Dougherty, W. G., and Carrington, J. C. (1988). Expression and function of potyviral gene products. Annu. Rev. Phytopathol. 26, 123-143. doi: 10.1146/annurev.py.26.090188.001011

\section{CONCLUSIONS}

This study provides a better way to computationally analyze the best-candidate miRNAs against viruses, prior to cloning. As our approach allows a narrow-range of match-mismatch in microRNA-mRNA attachment, it screens most of the falsely predicted attachments. The phylogenetic tree helps to determine whether there is any possibility for cloned miRNAs to act offtarget and affect other virus species. The more viral species that are discovered to be closer to $P V Y$, are more likely to hit by cloned miRNA.

\section{AUTHOR CONTRIBUTIONS}

The main idea was developed by MI and bioinformatics analysis were done by MI, MH, and AA, data interpretation was done by MS and JW and manuscript was written and proof-read jointly by all authors.

\section{SUPPLEMENTARY MATERIAL}

The Supplementary Material for this article can be found online at: http://journal.frontiersin.org/article/10.3389/fgene. 2016.00159

Eagles, R. M., Balmori-Melián, E., Beck, D. L., Gardner, R. C., and Forster, R. L. (1994). Characterization of NTPase, RNA-binding and RNA-helicase activities of the cytoplasmic inclusion protein of tamarillo mosaic potyvirus. Eur. J. Biochem. 224, 677-684. doi: 10.1111/j.1432-1033.1994.t01-100677.x

Edgar, R. C. (2004). MUSCLE: multiple sequence alignment with high accuracy and high throughput. Nucleic Acids Res. 32, 1792-1797. doi: 10.1093/nar/ gkh340

Fellers, J., Wan, J., Hong, Y., Collins, G. B., and Hunt, A. G. (1998). In vitro interactions between a potyvirus-encoded, genome-linked protein and RNAdependent RNA polymerase. J. Gen. Virology, 79, 2043-2049. doi: 10.1099/ 0022-1317-79-8-2043

Filipowicz, W., and Hohn, T. (eds.). (1996). Post-Transcriptional Control of Gene Expression in Plants. Kluwer Academic Publishers. doi: 10.1007/978-94-0090353-1

Glais, L., Tribodet, M., and Kerlan, C. (2002). Genomic variability in Potato potyvirus $\mathrm{Y}(P V Y)$ : evidence that $P V Y$ NW and $P V Y$ NTN variants are single to multiple recombinants between $P V Y \mathrm{O}$ and $P V Y \mathrm{~N}$ isolates. Arch. Virol. 147, 363-378. doi: 10.1007/s705-002-8325-0

Hariharan, M., Scaria, V., Pillai, B., and Brahmachari, S. K. (2005). Targets for human encoded microRNAs in HIV genes. Biochem. Biophys. Res. Commun. 337, 1214-1218. doi: 10.1016/j.bbrc.2005.09.183

Hong, Y., and Hunt, A. G. (1996). RNA polymerase activity catalyzed by a potyvirus-encoded RNA-dependent RNA polymerase. Virology 226, 146-151. doi: 10.1006/viro.1996.0639

John, B., Enright, A. J., Aravin, A., Tuschl, T., Sander, C., and Marks, D. S. (2004). Human microRNA targeteds. PLoS Biol. 2:e363. doi: 10.1371/journal. pbio. 0020363

Jones-Rhoades, M. W., Bartel, D. P., and Bartel, B. (2006). MicroRNAs and their regulatory roles in plants. Annu. Rev. Plant Biol. 57, 19-53. doi: 10.1146/ annurev.arplant.57.032905.105218

Karasev, A. V., and Gray, S. M. (2013). Genetic diversity of Potato virus Y complex. Am. J. Potato Res. 90, 7-13. doi: 10.1007/s12230-012-9287-7

Kasschau, K. D., and Carrington, J. C. (1998). A counter defensive strategy of plant viruses: suppression of posttranscriptional gene silencing. Cell 95, 461-470. doi: 10.1016/S0092-8674(00)81614-1 
Kim, V. N. (2005). MicroRNA biogenesis: coordinated cropping and dicing. Nat. Rev. Mol. Cell Biol. 6, 376-385. doi: 10.1038/nrm1644

Klein, P. G., Klein, R. R., Rodriguez-Cerezo, E., Hunt, A. G., and Shaw, J. G. (1994). Mutational analysis of the tobacco vein mottling virus genome. Virology 204, 759-769. doi: 10.1006/viro.1994.1591

Krüger, J., and Rehmsmeier, M. (2006). RNAhybrid: microRNA targeted prediction easy, fast and flexible. Nucleic Acids Res. 34, W451-W454. doi: $10.1093 / \mathrm{nar} / \mathrm{gkl} 243$

Lain, S., Riechmann, J. L., and Garcia, J. A. (1990). RNA helicase: a novel activity associated with a protein encoded by a positive strand RNA virus. Nucleic Acids Res. 18, 7003-7006. doi: 10.1093/nar/18.23.7003

Léonard, S., Plante, D., Wittmann, S., Daigneault, N., Fortin, M. G., and Laliberté, J. F. (2000). Complex formation between potyvirusVPg and translation eukaryotic initiation factor $4 \mathrm{E}$ correlates with virus infectivity. J. Virol. 74, 7730-7737. doi: 10.1128/JVI.74.17.7730-7737.2000

Li, X. H., Valdez, P., Olvera, R. E., and Carrington, J. C. (1997). Functions of the tobacco etch virus RNA polymerase (NIb): subcellular transport and protein-protein interaction with VPg/proteinase (NIa). J. Virol. 71, 1598-1607.

Liu, H. W., Luo, L. X., Liang, C. Q., Jiang, N., Liu, P. F., and Li, J. Q. (2015). Highthroughput sequencing identifies novel and conserved cucumber (Cucumis sativus $\mathrm{L}$.) microRNAs in response to cucumber green mottle mosaic virus infection. PLoS ONE 10:e0129002. doi: 10.1371/journal.pone.0129002

Lorenzen, J. H., Meacham, T., Berger, P. H., Shiel, P. J., Crosslin, J. M., Hamm, P. B., et al. (2006). Whole genome characterization of Potato virus $Y$ isolates collected in the western USA and their comparison to isolates from Europe and Canada. Arch. Virol. 151, 1055-1074. doi: 10.1007/s00705-005-0707-6

Mahajan, S., Dolja, V. V., and Carrington, J. C. (1996). Roles of the sequence encoding tobacco etch virus capsid protein in genome amplification: requirements for the translation process and a cis-active element. J. Virol. 70, 4370-4379.

McCue, K. F., Ponciano, G., Rockhold, D. R., Whitworth, J. L., Gray, S. M., Fofanov, Y., et al. (2012). Generation of PVY Coat Protein siRNAs in Transgenic Potatoes Resistant to PVY. Am. J. Potato Res. 89, 374-383. doi: 10.1007/s12230012-9257-0

Merits, A., Guo, D., and Saarma, M. (1998). VPg, coat protein and five nonstructural proteins of potato Apotyvirus bind RNA in a sequence-unspecific manner. J. Gen. Virol. 79, 3123-3127. doi: 10.1099/0022-1317-79-12-3123

Miranda, K. C., Huynh, T., Tay, Y., Ang, Y. S., Tam, W. L., Thomson, A. M., et al. (2006). A pattern-based method for the identification of MicroRNA binding sites and their corresponding heteroduplexes. Cell 126, 1203-1217. doi: 10.1016/j.cell.2006.07.031

Missiou, A., Kalantidis, K., Boutla, A., Tzortzakaki, S., Tabler, M., and Tsagris, M. (2004). Generation of transgenic potato plants highly resistant to potato virus Y $(P V Y)$ through RNA silencing. Mol. Breed. 14, 185-197. doi: 10.1023/B:MOLB. 0000038006.32812 .52

Mlotshwa, S., Pruss, G. J., and Vance, V. (2008). Small RNAs in viral infection and host defense. Trends Plant Sci. 13, 375-382. doi: 10.1016/j.tplants.2008.04.009

Pillai, R. S., Bhattacharyya, S. N., and Filipowicz, W. (2007). Repression of protein synthesis by miRNAs: how many mechanisms? Trends Cell Biol. 17, 118-126. doi: 10.1016/j.tcb.2006.12.007

Pirone, T. P., and Thornbury, D. W. (1984). The involvement of a helper component in nonpersistent transmission of plant viruses by aphids. Microbiol. Sci. 1, 191-193.

Rajamäki, M. L., and Valkonen, J. P. (1999). The 6K2 protein and the VPg of potato virus A are determinants of systemic infection in Nicandraphysaloides. Mol. Plant Microbe Interact. 12, 1074-1081. doi: 10.1094/MPMI.1999.12.12.1074

Restrepo-Hartwig, M. A., and Carrington, J. C. (1994). The tobacco etch potyvirus 6-kilodalton protein is membrane associated and involved in viral replication. J. Virol. 68, 2388-2397.

Riechmann, J. L., Laín, S., and García, J. A. (1992). Highlights and prospects of potyvirus molecular biology. J. Gen. Virol. 73, 1-16. doi: 10.1099/0022-131773-1-1
Robert, Y., Woodford, J. T., and Ducray-Bourdin, D. G. (2000). Some epidemiological approaches to the control of aphid-borne virus diseases in seed potato crops in northern Europe. Virus Res. 71, 33-47. doi: 10.1016/S01681702(00)00186-6

Rogers, K., and Chen, X. (2013). Biogenesis, turnover, and mode of action of plant microRNAs. Plant Cell 25, 2383-2399. doi: 10.1105/tpc.113.113159

Rojas, M. R., Zerbini, F. M., Allison, R. F., Gilbertson, R. L., and Lucas, W. J. (1997). Capsid protein and helper component-proteinase function as potyvirus cell-tocell movement proteins. Virology 237, 283-295. doi: 10.1006/viro.1997.8777

Rovere, C. V., Del Vas, M., and Hopp, H. E. (2002). RNA-mediated virus resistance. Curr. Opin. Biotechnol. 13, 167-172. doi: 10.1016/S0958-1669(02)00296-3

Ruffel, S., Dussault, M. H., Palloix, A., Moury, B., Bendahmane, A., Robaglia, C., et al. (2002). A natural recessive resistance gene against potato virus $Y$ in pepper corresponds to the eukaryotic initiation factor 4E (eIF4E). Plant J. 32, 1067-1075. doi: 10.1046/j.1365-313X.2002.01499.x

Schaad, M. C., Haldeman-Cahill, R., Cronin, S., and Carrington, J. C. (1996). Analysis of the VPg-proteinase (NIa) encoded by tobacco etch potyvirus: effects of mutations on subcellular transport, proteolytic processing, and genome amplification. J. Virol. 70, 7039-7048.

Shukla, D. D., and Ward, C. W. (1989). Structure of potyvirus coat proteins and its application in the taxonomy of the potyvirus group. Adv. Virus Res. 36, 273-314. doi: 10.1016/S0065-3527(08)60588-6

Srivastava, P. K., Moturu, T. R., Pandey, P., Baldwin, I. T., and Pandey, S. P. (2014). A comparison of performance of plant miRNA target prediction tools and the characterization of features for genome-wide target prediction. BMC Genomics 15:348. doi: 10.1186/1471-2164-15-348

Tabassum, B., Nasir, I. A., and Husnain, T. (2011). Potato virus Y mRNA expression knockdown mediated by siRNAs in cultured mammalian cell line. Virol. Sin. 26, 105-113. doi: 10.1007/s12250-011-3161-x

Teycheney, P. Y., Aaziz, R., Dinant, S., Salánki, K., Tourneur, C., Balázs, E., et al. (2000). Synthesis of (-)-strand RNA from the $3^{\prime}$ untranslated region of plant viral genomes expressed in transgenic plants upon infection with related viruses. J. Gen. Virol. 81, 1121-1126. doi: 10.1099/0022-1317-81-4-1121

Valencia-Sanchez, M. A., Liu, J., Hannon, G. J., and Parker, R. (2006). Control of translation and mRNA degradation by miRNAs and siRNAs. Genes Dev. 20, 515-524. doi: 10.1101/gad.1399806

Verchot, J., Koonin, E. V., and Carrington, J. C. (1991). The 35-kDa protein from the $\mathrm{N}$-terminus of the potyviralpolyprotein functions as a third virus-encoded proteinase. Virology 185, 527-535. doi: 10.1016/0042-6822(91)90522-D

Witkos, T. M., Koscianska, E., and Krzyzosiak, W. J. (2011). Practical aspects of microRNA target prediction. Curr. Mol. Med. 11, 93-109. doi: $10.2174 / 156652411794859250$

Wittmann, S., Chatel, H., Fortin, M. G., and Laliberté, J. F. (1997). Interaction of the viral protein genome linked of turnip mosaic potyvirus with the translational eukaryotic initiation factor (iso) $4 \mathrm{E}$ of Arabidopsis thaliana using the yeast two-hybrid system. Virology 234, 84-92. doi: 10.1006/viro.1997.8634

Zhu, C. X., Song, Y. Z., Yin, G. H., and Wen, F. J. (2009). Induction of RNA-mediated Multiple Virus Resistance to Potato virus Y, Tobacco mosaic virus and Cucumber mosaic virus. J. Phytopathol. 157, 101-107. doi: 10.1111/j.1439-0434.2008.01449.x

Conflict of Interest Statement: The authors declare that the research was conducted in the absence of any commercial or financial relationships that could be construed as a potential conflict of interest.

Copyright $\odot 2016$ Iqbal, Hafeez, Wattoo, Ali, Sharif, Rashid, Tabassum and Nasir. This is an open-access article distributed under the terms of the Creative Commons Attribution License (CC BY). The use, distribution or reproduction in other forums is permitted, provided the original author(s) or licensor are credited and that the original publication in this journal is cited, in accordance with accepted academic practice. No use, distribution or reproduction is permitted which does not comply with these terms. 